\title{
The hypothesis of the toxic effects of the venous collateral circulation
}

\section{F Passariello 1}

${ }^{1}$ Fondazione Vasculab ONLUS, via Francesco Cilea 280 - 80127 Naples, Italy

Contribute to a Vasculab discussion in July 2013

submitted: Sep 20, 2017, accepted: Sep 25, 2017, EPub Ahead of Print: Sep 26, 2017, published: Sep 27, 2017

Conflict of interest: None

DOI: 10.24019/jtavr.34 - Corresponding author: Dr. Fausto Passariello, afunzionale@tiscalinet.it

(C) 2017 Fondazione Vasculab impresa sociale ONLUS. All rights reserved.

Abstract Comparing the arterial and venous collateral circulation interesting symmetries are detected as well as important differences. Venous compensating pathways are essentially intra-parenchymal and convey dirty as deoxygenated blood. The hypothesis of the toxic effects of the venous collateral circulation is formulated, i.e. the greater the volume of compensation, the greater the toxic damage. Two scenarios are previewed as possible targets, i.e. the postthrombotic syndrome (PTS) and the chronic cerebrovascular insufficiency (CCSVI).

Keywords Blood collateral circulation, postthrombotic syndrome, MS,CCSVI.

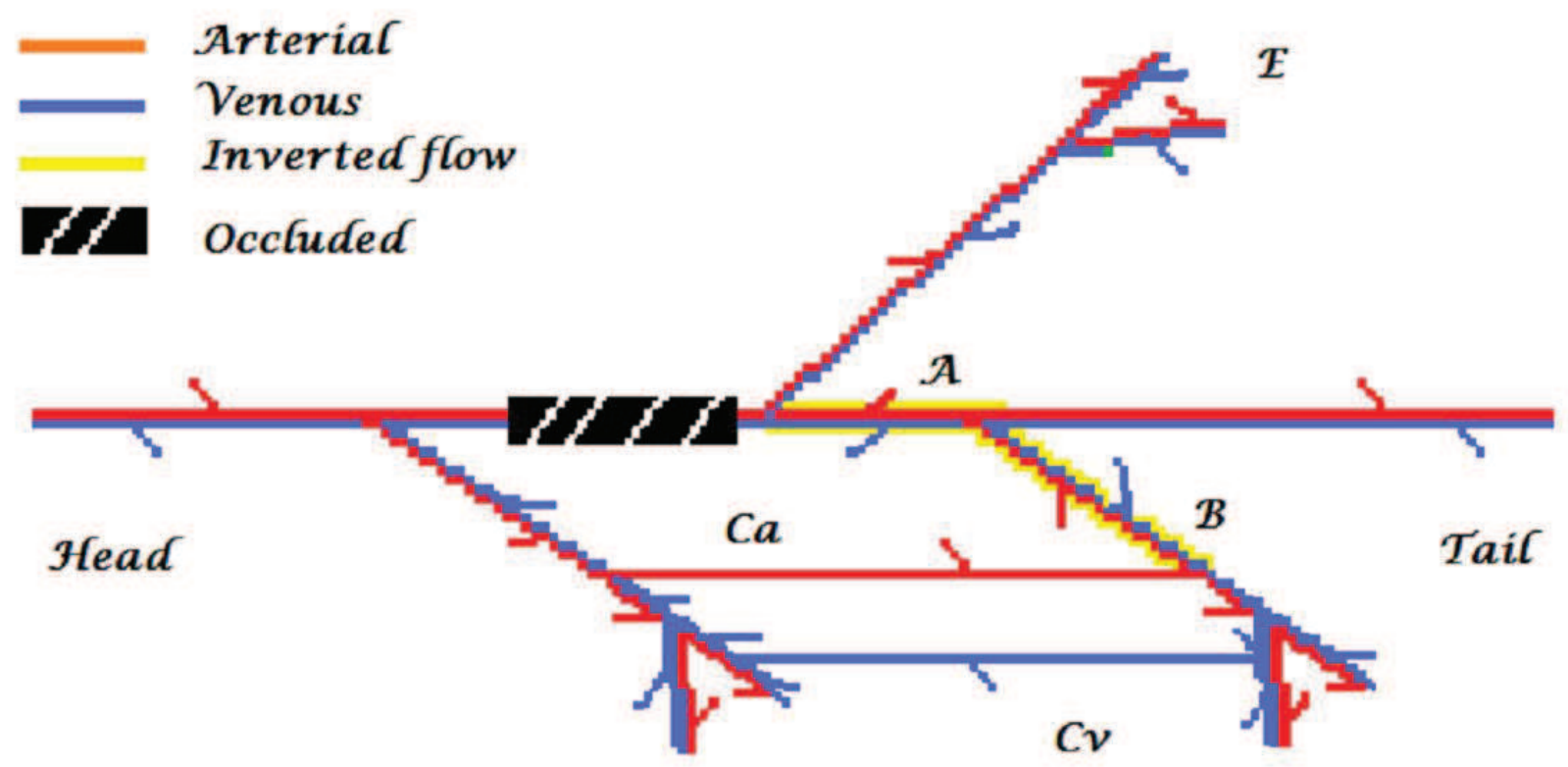

Figure 1 - Collateral circulation by-passing an obstruction in an arterial and venous schematic example. Half arrows point to the direction of flow, red for arteries, blue for veins. Yellow for inverted flow. Black for occlusion. A, inverted main trunk. B, inverted collateral path. E, excluded region. Ca, arterial anastomosis. $C v$, venous anastomosis. 


\section{Collateral circulation in arteries and veins}

$\begin{array}{lll} & \text { Arterial } & \text { Venous } \\ \begin{array}{l}\text { Collateral circulation } \\ \text { Speed }\end{array} & \begin{array}{l}\text { Transport vessels } \\ \text { High }\end{array} & \begin{array}{l}\text { Even small peripheral veins } \\ \text { Low }\end{array} \\ \text { Main trunk inversion } & & \text { Valve incompetence, if any } \\ \text { Profile } & \text { Blunt } & \text { Parabolic } \\ \text { Blood } & \text { Clean and Oxygenated } & \text { Dirty and Deoxygenate } \\ \text { Wall } & \text { Stiffer } & \text { Collapsible } \\ \text { Shear rate } & \text { High } & \text { Low }\end{array}$

Table 1 - Morphological and hemodynamic differences in post-thrombotic collateral circulation in the lower limbs.

An interesting discussion on the venous collateral circulation was held on Vasculab in July $2013^{1}$. The following lines report a theory which was formulated about.

Compensating vessels in the venous system follow essentially different patterns than in the arterial system. (Fig. 1)

\section{Arteries}

a. The arterial tree (red) is designed with a complete occlusion and a collateral circulation which jumps the occlusion, where flow runs from the Head to the Tail of the picture. (e.g. occlusion of the femoral artery, compensated through the deep femoral artery and a Hunter arterial perforator)

b. There must be a previous natural or newly formed arterial anastomosis $(\mathrm{Ca})$ between the 2 main components of the compensating path.

c. In addition, the (B) component is inverted, in comparison with the physiological flow and the same occurs for the main trunk (A), as it carries a feeding flow towards an excluded region (E). Inverted arteries are contoured by a yellow colour.

\section{Veins}

a. The same picture can serve to represent the venous tree (blue) with a complete occlusion and a collateral circulation which jumps the occlusion, where now flow runs from the Tail to the Head of the picture. (e.g. occlusion of the femoral vein, compensated through the deep femoral vein and a Hunter venous perforator)

b. There must be a previous natural or newly formed venous anastomosis $(\mathrm{Cv})$ between the 2 main components of the compensating path.

c. In addition, the (B) component is inverted, in comparison with the physiological flow and the same occurs for the main trunk (A), as it carries a drainage flow coming from an excluded region (E). Inverted veins are contoured by a yellow colour.

In the depicted structure there is an important symmetry in structure and function, which depends on the designed scheme and the direction of flow.
However this symmetry is abandoned as soon as we introduce some specific venous structures.

The A venous tract can contain valves, thus the inverted flow can occur only if the valve is destroyed of forced to be incompetent. This is of course achieved by the hypertension caused by an impaired venous outflow.

In addition, a lot of differences can be found and are reported in Table 1.

The main remarks are:

1) The Energy density (Energy per unit volume) is

$\frac{E}{V}=p+\rho g h+\frac{1}{2} \rho v^{2}$

In veins, the mean velocity is generally much smaller than in arteries, thus phenomena are governed by hydrostatics much more than hydrodynamics, i.e. the energy density is almost the same than in a static container.

Thus, whatever the stenosis/occlusion in the system, whatever the posture, as soon as the blood by-passes it, just downstream the obstacle the pressure is almost very near to the upstream value, i.e. the pressure difference is minimal.

2) An hypothesis can be proposed, that the venous collateral circulation is generally spread in more peripheral vessels than in arteries, i.e. in veins, the collateral circulation can be intraparenchymal. The hypothesis is partially justified looking at the A segment in Fig. 1, which is caudal to the occlusion in the artery, cranial to the occlusion in the vein, in the same tree-like structure.

3) The venous collateral circulation conveys dirty and deoxygenated blood, just the contrary than in arteries. This does not include arterial-venous fistulas, where veins receive oxygenated blood.

- Tissues which are overcharged by collateral arteries receive oxygen and are fed by them.

- Tissues which are overcharged by collateral veins receive catabolites and carbon dioxide. 
There is enough material to formulate the theory of the Toxic Effects of the Venous Collateral Circulation. (TEVCC)

Two scenarios are previewed as possible targets:

- the lower limb veins, where the effect is the same than in chronic venous insufficiency, when the closed shunts convey back dirty as deoxygenated blood. However, the most immediate recall is to the postthrombotic syndrome (PTS), with its huge and long lasting tissue damages;

- the venous cerebrospinal circulation in multiple sclerosis (MS) instead, where the clinical presentation is the chronic cerebrovascular insufficiency (CCSVI).

As regards CCSVI, the TEVCC hypothesis can be interestingly reformulated as follows:

Stenosis/occlusion in the venous cerebrospinal system causes a venous surcharge by collateral circulation inside the brain and the spinal cord system.
The hypothesis suggests that it could have an effect only when a valid collateral circulation has already developed.

The greater the volume of compensation, the greater the toxic damage.

\section{Conclusions}

As a consequence the pathology could be related not only to the severity of the stenosis/occlusion, but also to the exuberance of the collateral circulation with its toxic effects, i.e. to the TEVCC.

As regards MS, the hypothesis could be easily tested looking at the distribution in clinical severity of MS of the magnetic resonance arterial/venous flow mismatch (AVM), interpreted as the amount of outflow through small or collateral veins ${ }^{2,3}$.

\section{Acknowledgements}

I thank Mrs. Iolanda Palma for her invaluable help in typesetting the manuscript.

Flow Distribution of the Internal Jugular Veins. J Vasc Interv Radiol 2012; 23:60\#68. DOI: 10.1016/j.jvir.2011.09.027.

3) Passariello F. Review of the article "Patients with Multiple Sclerosis with Structural Venous Abnormalities on MR Imaging Exhibit an Abnormal Flow Distribution of the Internal Jugular Veins" by Haacke EM. Phlebology Forum 2012; May-June:15-18. Accessed at http://www.phlebology.org/memberresources/publications/phlebology-forum on Sep 26, 2017. 